\title{
Modified totally tubeless percutaneous nephrolithotomy: Is it an effective and safe treatment option for renal and upper ureteral stones?
}

\author{
Ho Seok Chung, Seung Il Jung, Ho Song Yu, Eu Chang Hwang, Kyung Jin Oh, Dong Deuk Kwon, Kwangsung Park \\ Department of Urology, Medical School, Chonnam National University, Gwangju, Korea \\ Videosurgery Miniinv 2016; 11 (4): 240-246 \\ DOI: https://doi.org/10.5114/wiitm.2016.64447
}

\begin{abstract}
Introduction: We hypothesized that modified totally tubeless percutaneous nephrolithotomy (PNL) without indwelling ureteral stent would minimize postoperative discomfort without complications.

Aim: To evaluate the safety, efficacy, and morbidity of standard, tubeless, and modified totally tubeless PNL as well as the usefulness of modified totally tubeless PNL.

Material and methods: From November 2011 to February 2015, 211 patients who underwent PNL consecutively were enrolled in this study and divided into 3 groups (group 1: standard, group 2: tubeless, group 3: modified totally tubeless PNL). Patient and stone characteristics, operation time, hemoglobin change, length of hospitalization, stonefree rate, analgesic requirement, and perioperative complications were analyzed and compared among the 3 groups. Results: There were no significant differences in preoperative patient characteristics among the three groups. In the postoperative analysis, the three groups had similar operation time, stone-free rate, perioperative fever and transfusion rate, but group 2 showed superior results in terms of length of hospitalization $(p=0.001)$. Group 2 and group 3 had a lower analgesic requirement $(p=0.010)$. Immediate postoperative hemoglobin change $(p=0.001)$ and tube site complications ( $p=0.001)$ were more common in group 1 .

Conclusions: Modified totally tubeless PNL was not inferior in terms of postoperative outcomes and safety compared with the standard and tubeless PNL, and avoided the postoperative stent-related symptoms and cystoscopy for double-J stent removal. Modified totally tubeless PNL could be an alternative treatment of choice for management of renal or upper ureteral stones in selected patients.
\end{abstract}

Key words: percutaneous nephrolithotomy, stone, tubeless.

\section{Introduction}

Percutaneous nephrolithotomy (PNL) has been widely used to remove large and complex renal stones since the first PNL was described in 1976 [1]. The placement of a nephrostomy catheter at the end of the PNL has been considered the standard procedure, which is thought to act as a tamponade to stop bleeding, provide adequate renal drainage, and make an additional nephroscopy easier. Despite these advantages, in recent years, nephrostomy catheters have been thought to be associated with prolonged hospitalization, urine extravasation, and significant postoperative pain and discomfort [2]. For this reason, a modified technique using external and internal ureteral stents instead of nephrostomy catheters for postoperative drainage after PNL was performed to reduce catheter-related morbidity, and it is known as tubeless PNL [3-5]. Also, some studies

\section{Address for correspondence}

Seung II Jung, Department of Urology, Medical School, Chonnam National University, Hwasun Hospital, 322 Seoyang-ro, Hwasun 519-763, Republic of Korea, phone: +82 61379 8160, fax: +82 61379 7750, e-mail: drjsi@yahoo.co.kr 
have reported about a technique that does not use a nephrostomy catheter and ureteral stent after PNL, and it is called totally tubeless PNL [6-8].

\section{Aim}

We hypothesized that the placement of an external $5 \mathrm{Fr}$ ureteral stent below the renal pelvis after the PNL without a double-J stent, modified totally tubeless PNL would provide adequate drainage of the kidney and minimize postoperative discomfort without complications. In this study, we evaluated the safety, efficacy, and morbidity of standard, tubeless, and modified totally tubeless PNL in a single center as well as the usefulness of modified totally tubeless PNL.

\section{Material and methods}

\section{Patients}

We retrospectively evaluated the records of 211 patients who underwent PNL for the treatment of renal or upper ureteral stones at our institution consecutively from November 2011 to February 2015. We divided the patients into 3 groups (group 1: standard PNL, group 2: tubeless PNL, group 3: externalized ureteral stent, named modified totally tubeless PNL) and performed PNL according to our preference (Photo 1). Standard and modified totally tubeless PNL were considered for second-look endoscopic procedures according to intraoperative findings. Laboratory tests were performed before the surgery including complete blood count, creatinine levels, and urine culture. Radiological evaluation was performed with plain radiography, ultrasonography, and computed tomography.

\section{Operative procedures}

Under general anesthesia, a retrograde $5 \mathrm{Fr}$ ureteral catheter was placed into the upper ureter with the aid of a cystoscope and then the catheter was attached to the urethral Foley catheter in the supine position. The patient was then placed in the prone position, and percutaneous access to the selected calyx was obtained under C-arm fluoroscopic guidance using a 21-gauge access needle. After entering the collecting system with a guide wire, dilatation was performed using a balloon dilator for the passage of the working sheath. Through the working sheath, a $26 \mathrm{Fr}$ rigid nephroscope was inserted into the renal collecting system, stone fragmentation was carried out with a pneumatic lithotripter and stone fragments were extracted with graspers. At the end of the surgery, fluoroscopy and pyelography were performed for evaluation of the collecting system to assess the stone-free status and to identify the patients with clinically insignificant residual fragments $(<4 \mathrm{~mm})$.

After completion of the PNL, a $24 \mathrm{Fr}$ Malecot catheter was placed in patients of group 1, and an antegrade $6 \mathrm{Fr}$ double-J stent was inserted, but no nephrostomy catheter was placed in patients of group 2 . In patients of group 3, an externalized $5 \mathrm{Fr}$ ureteral stent was inserted with nephroscopy and fluoroscopy, the tip being placed in the proximal or mid ureter. The incision of the nephrostomy tracts was closed with silk sutures and hemostatic agents were not used. After the surgery, supine radiography or ultrasonography or computed tomography of the kidneys, ureters, and bladder was performed in each patient to assess stone clearance within 2 weeks after surgery. The postoperative stone free status was defined as no stones visible or residual fragments $<4 \mathrm{~mm}$ on the postoperative images. Patients were given tramadol $\mathrm{HCl}$ (non-narcotic analgesic) to control postoperative pain.

The decision of nephrostomy catheter removal in group 1 was made based on the urine color, and the nephrostomy tract was closed with silk sutures immediately after catheter removal. Double-J stents placed in patients of group 2 were removed 2 weeks after the surgery with cystoscopy, and only the Foley catheters were removed the day after surgery. In group 3 , the externalized ureteral stent and Foley catheter were removed simultaneously the day after surgery. Serum hemoglobin was measured immediately after the operation and on the first day after surgery.

The three groups were compared with respect to patient and stone characteristics, operative time, postoperative hemoglobin change, length of hospitalization, blood transfusion rate, analgesic need, stone-free rate, and perioperative complications such as hydrothorax and urinary leak. Stone burden was measured by using the largest width and length obtained from the plain radiograph or computed tomography. The follow-up evaluations were performed at 2 weeks postoperatively, and assessments were made with routine laboratory tests, urine culture, and clinical examination. This study protocol was reviewed and approved by the institutional review board. Informed consent was waived by the board. 

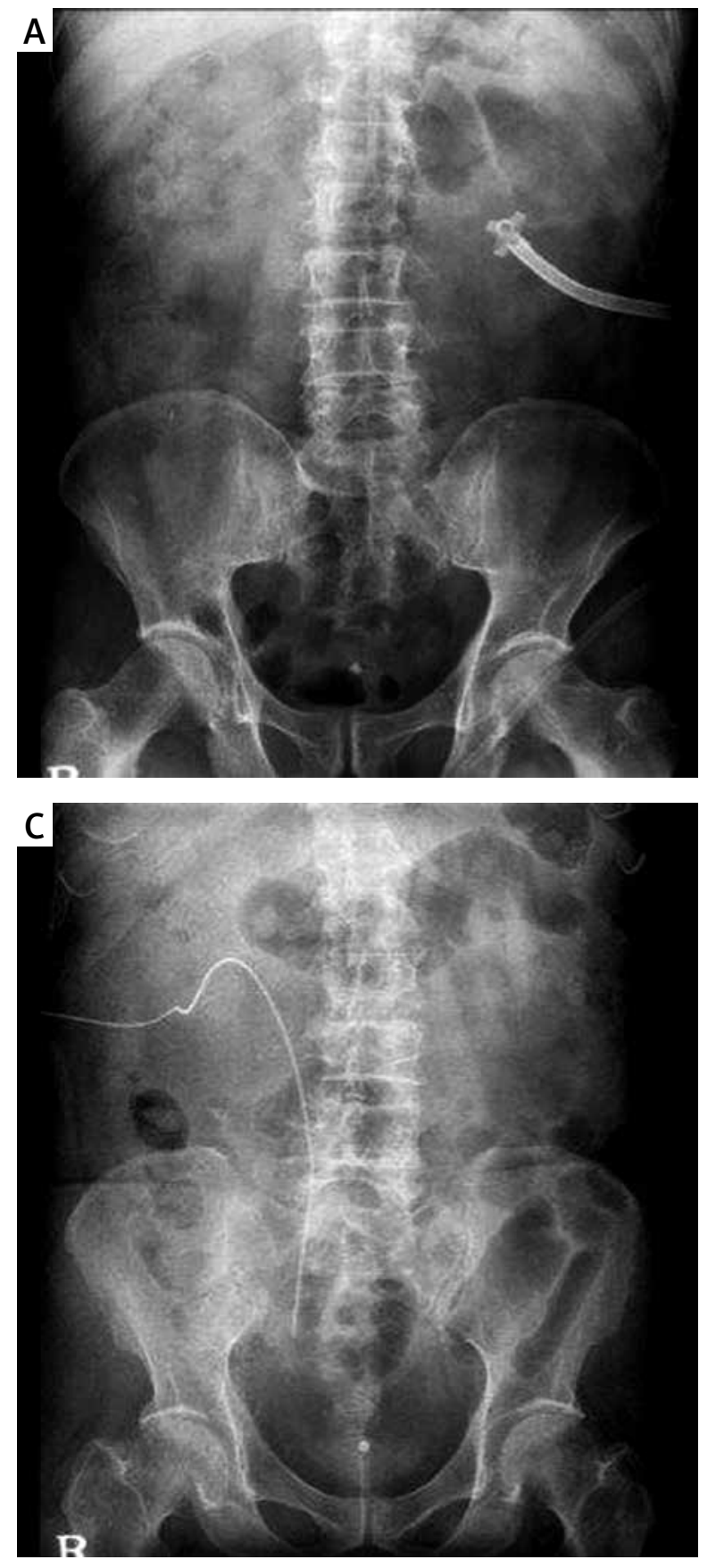

\section{Statistical analysis}

Statistical analyses were performed with the SPSS software ver. 19.0 (SPSS Inc., Chicago, IL, USA). Continuous data are presented as mean \pm SD, and discrete categorical variables are presented as a number (\%). The Kruskal-Wallis and $\chi^{2}$ tests, and Tukey HSD post hoc analysis were performed to compare the differences in clinical variables among

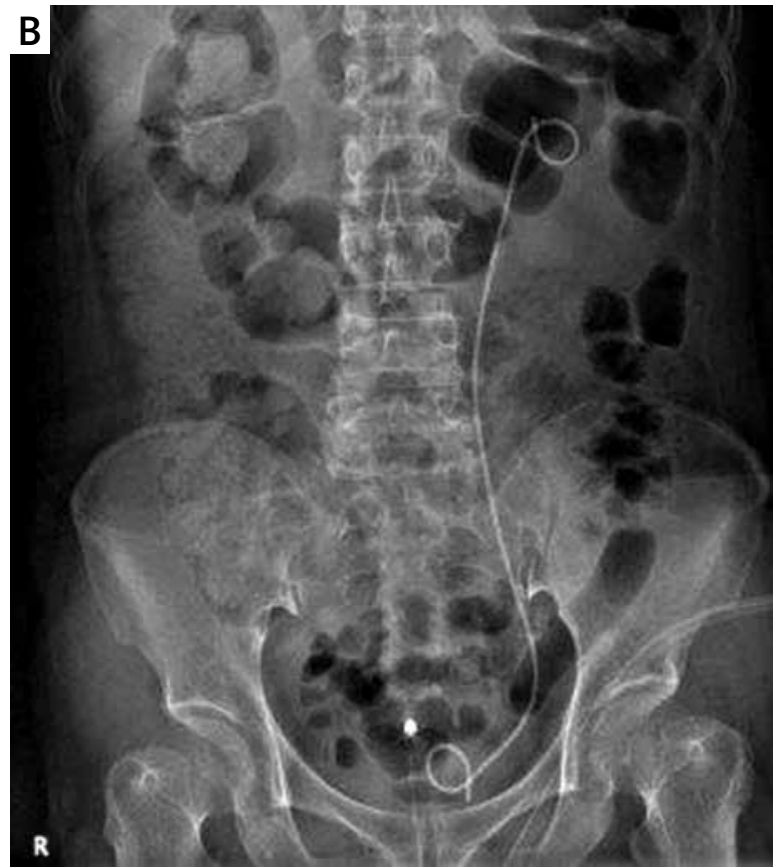

Photo 1. Technique with PNL. A - Standard PNL (group 1), B - Tubeless PNL (group 2), C - Modified totally tubeless PNL (group 3)

the three groups. $P$-values less than 0.05 were considered statistically significant.

\section{Results}

A total of 211 patients who underwent PNL consecutively for the treatment of renal stones, with or without upper ureteral stones, were included in our study. Among these, 42 patients underwent 
standard PNL (group 1), 108 patients underwent tubeless PNL (group 2), and 61 patients underwent modified totally tubeless PNL (group 3). There were no significant differences in the preoperative patient characteristics such as age, sex, body mass index (BMI), operation site, stone size, location, multiplicity and bacterial pyuria among the 3 groups (Table I).

Postoperative analyses in each group are summarized in Table II. No significant differences were found in the operation time, perioperative stonefree rate assessed with a plain radiograph, transfusion rate, and perioperative fever episodes among the 3 groups $(p>0.05)$. Length of hospitalization and nephrostomy site stitch removal was shorter in group 2 ( $p=0.001)$ compared with the other groups. Immediate postoperative hemoglobin change, and analgesic requirement and dosage were found to be significantly higher in group 1 (immediate postoperative hemoglobin change, $p=0.001$; analgesic requirement, $p=0.010$; analgesic dose, $p=0.001$ ).
Nephrostomy catheter site complications including wound dehiscence and remnant tract with urine leakage were more common in group $1(p=0.001)$.

There were no adjacent organ injuries during the surgery in the 3 groups. However, 2 patients in group 1 and 1 patient in group 2 with hematuria were diagnosed as having a pseudoaneurysm on renal angiography. These 3 patients underwent selective renal angioembolization to control bleeding. Two patients in group 3 developed spontaneous migration of small remnant stone fragments with flank pain immediately after discharge and were readmitted to the hospital and treated conservatively.

\section{Discussion}

In our study, tubeless PNL and modified totally tubeless PNL had benefits in terms of safety and postoperative outcomes compared with standard PNL. Tubeless PNL had shorter hospitalization and lower postoperative analgesic requirement. Modified

Table I. Characteristics of patients and stones

\begin{tabular}{|c|c|c|c|c|}
\hline Parameter & Group $1(n=42)$ & Group $2(n=108)$ & Group $3(n=61)$ & $P$-value \\
\hline Age & $58.8 \pm 13.2$ & $54.5 \pm 12.6$ & $56.6 \pm 13.2$ & $0.161^{*}$ \\
\hline \multicolumn{5}{|l|}{ Gender: } \\
\hline Male & $22(52.4)$ & $63(58.3)$ & $38(62.3)$ & \multirow[t]{2}{*}{$0.608^{\dagger}$} \\
\hline Female & $20(47.6)$ & $45(41.7)$ & $23(37.7)$ & \\
\hline BMI $\left[\mathrm{kg} / \mathrm{m}^{2}\right]$ & $25.1 \pm 3.3$ & $24.9 \pm 3.4$ & $24.7 \pm 4.0$ & $0.920^{*}$ \\
\hline \multicolumn{5}{|l|}{ Operation site: } \\
\hline Right & $16(38.1)$ & 45 (41.6) & $23(37.7)$ & \multirow[t]{2}{*}{$0.854^{\dagger}$} \\
\hline Left & $26(61.9)$ & $63(58.4)$ & $38(62.3)$ & \\
\hline \multicolumn{5}{|l|}{ Stone location: } \\
\hline Renal stone & $16(38.1)$ & $59(54.6)$ & $37(60.6)$ & \multirow[t]{4}{*}{$0.396^{\dagger}$} \\
\hline Staghorn stone & $20(47.6)$ & $39(36.1)$ & $14(23.0)$ & \\
\hline Ureteric stone & $3(7.1)$ & $4(3.7)$ & $3(4.9)$ & \\
\hline Renal and ureteric stone & $3(7.1)$ & $7(6.4)$ & $6(9.8)$ & \\
\hline \multicolumn{5}{|l|}{ Multiplicity: } \\
\hline Single & $26(61.9)$ & $53(49.1)$ & $28(45.9)$ & \multirow[t]{2}{*}{$0.251^{\dagger}$} \\
\hline Multiple & $16(38.1)$ & $55(50.9)$ & $33(54.1)$ & \\
\hline Stone size $[\mathrm{cm}]$ & $4.5 \pm 1.3$ & $4.2 \pm 1.4$ & $4.2 \pm 1.1$ & $0.557^{*}$ \\
\hline Bacterial pyuria & $5(11.9)$ & $13(12.0)$ & $12(19.7)$ & $0.351^{\dagger}$ \\
\hline
\end{tabular}

$B M I-$ body mass index. ${ }^{*} K r u s k a l-$ Wallis test, ${ }^{\dagger} \chi^{2}$ test. 
Table II. Comparison of operative and postoperative results

\begin{tabular}{|c|c|c|c|c|}
\hline Parameter & Group $1(n=42)$ & Group $2(n=108)$ & Group $3(n=61)$ & $P$-value \\
\hline Operation time [min] & $108.1 \pm 31.7$ & $113.8 \pm 24.1$ & $104.1 \pm 34.4$ & 0.102 \\
\hline Preoperative Hgb [g/dl] & $13.6 \pm 1.6$ & $13.4 \pm 1.8$ & $13.4 \pm 2.0$ & $0.845^{*}$ \\
\hline \multicolumn{5}{|l|}{ Decrease in $\mathrm{Hgb}[\mathrm{g} / \mathrm{dl}]$ : } \\
\hline Immediate postoperative $\mathrm{Hgb}$ & $11.3 \pm 1.5$ & $11.9 \pm 1.8$ & $11.9 \pm 1.9$ & $0.141^{*}$ \\
\hline POD \#1 Hgb & $11.0 \pm 1.4$ & $11.5 \pm 1.7$ & $11.7 \pm 1.9$ & $0.091^{*}$ \\
\hline Preoperative - immediate postoperative Hgb & $2.3 \pm 1.3^{a}$ & $1.4 \pm 1.1^{\mathrm{a}}$ & $1.5 \pm 1.1^{\mathrm{a}}$ & $0.001^{*}$ \\
\hline Immediate postoperative - POD \#1 Hgb & $0.3 \pm 1.1$ & $0.5 \pm 0.9$ & $0.2 \pm 0.9$ & 0.174 \\
\hline Length of hospitalization [days] & $6.8 \pm 2.9^{b}$ & $5.2 \pm 1.6^{b}$ & $5.9 \pm 2.7^{b}$ & $0.001^{*}$ \\
\hline \multicolumn{5}{|l|}{ Episodes of fever: } \\
\hline None & $19(45.2)$ & $68(63.0)$ & $41(67.2)$ & \multirow[t]{3}{*}{$0.336^{\dagger}$} \\
\hline Mild $\left(37.0-37.9^{\circ} \mathrm{C}\right)$ & $17(40.5)$ & $32(29.6)$ & $15(24.6)$ & \\
\hline $\operatorname{High}\left(\geq 38.0^{\circ} \mathrm{C}\right)$ & $6(14.3)$ & $8(7.4)$ & $5(8.2)$ & \\
\hline Transfusion rate (\%) & $10(23.8)$ & $9(8.3)$ & $13(21.3)$ & $0.052^{\dagger}$ \\
\hline Stone-free rate (\%) & $30(71.4)$ & $91(84.2)$ & $49(80.3)$ & $0.208^{+}$ \\
\hline \multicolumn{5}{|l|}{ Nephrostomy site complication: } \\
\hline None & $21(50.0)$ & $102(94.4)$ & $49(80.3)$ & \multirow[t]{3}{*}{$0.001^{\dagger}$} \\
\hline Complication & $9(21.4)$ & $2(1.9)$ & $9(14.8)$ & \\
\hline Simple oozing & $12(28.6)$ & $4(3.7)$ & $3(4.9)$ & \\
\hline Nephrostomy site stitch removal [days] & $14.3 \pm 4.6^{c}$ & $8.4 \pm 3.0^{c}$ & $9.3 \pm 2.8^{c}$ & $0.001^{*}$ \\
\hline \multicolumn{5}{|l|}{ Analgesic: } \\
\hline Analgesic requirement (\%) & $26(61.9)$ & $35(32.4)$ & $25(41.0)$ & $0.010^{\dagger}$ \\
\hline Analgesic dose [mg] & $130.5 \pm 177.0^{d}$ & $33.7 \pm 75.0^{d}$ & $29.8 \pm 59.2^{d}$ & $0.001^{*}$ \\
\hline
\end{tabular}

totally tubeless PNL could avoid ureteral stent-related symptoms, and postoperative cystoscopy to remove the double-J stent.

Percutaneous nephrostomy for treatment of hydronephrosis was described in 1955 [9], but removal of stones via the percutaneous access could not be accomplished. In 1976, PNL was first performed by Fernström and Johansson [1] and has been widely used to treat large and complex renal stones. The standard PNL includes the placement of a large-caliber nephrostomy tube at the end of the PNL that acts as a tamponade to stop bleeding, provides adequate renal drainage, and makes an additional endoscopic procedure easier. But despite these obvious advantages, nephrostomy tubes are thought to be associated with prolonged hospitalization, urine extravasation, and postoperative pain and discomfort [2]. Similarly, in our experience, patients who underwent standard PNL showed a higher analgesic requirement and more frequent nephrostomy tube site complications, including wound dehiscence and urine leakage, compared with patients in the other groups. Also, the length of hospitalization and nephrostomy site stitch removal were also longer in the standard PNL group. It could be affected by the transfusion rate, delayed nephrostomy tract closure 
after nephrostomy catheter removal and more frequent nephrostomy site complications.

In recent years, several modifications of PNL have been tried to decrease the length of hospitalization, postoperative morbidity and pain. Wickham et al. [10] first introduced the totally tubeless PNL in 1984 without external and internal ureteral stents, but it was not well used in the following years. Thereafter, Bellman et al. [11] reported the tubeless PNL with only a double-J stent for internal drainage, which decreased the length of hospitalization, postoperative analgesic requirement, and cost. After this report, the tubeless PNL gained popularity and subsequent case series confirmed the efficacy of the procedure [12-15]. Al-Ba'adani et al. [16] emphasized that tubeless PNL is the new gold standard. They stated that in 121 patients, tubeless PNL was effective and insertion of a double-J stent was sufficient for renal drainage, reaching a complication rate of $9.9 \%$. In our study, tubeless PNL performed in 73 patients showed similar results including shorter length of hospitalization, and lower analgesic requirement and nephrostomy tube site complications.

However, patients who underwent tubeless PNL have stent-related symptoms and postoperative cystoscopy should be performed for stent removal. Shah et al. [3] reported that $30 \%$ of the patients who underwent tubeless PNL had discomfort related to the double-J stent, and $60 \%$ of these patients needed medication for symptom relief. Agrawal et al. [17] reported that after tubeless PNL using an antegrade tether, the stent can be removed directly by pulling the attached tether in the office setting at 2 weeks postoperatively. They stated that it avoids the need for cystoscopy for stent removal and provides access for the second-look nephroscopy, but stent-related discomfort could not be avoided.

These shortcomings led to modifications, and Karami and Gholamrezaie [18] compared 30 totally tubeless PNL cases with 30 standard PNL cases. They stated that avoiding the use of a nephrostomy tube and ureteral stent at the end of the surgery in selected patients was a safe option, and they observed significantly decreased length of hospitalization and analgesic requirement. Also, Crook et al. [6] reported that randomly performed totally tubeless PNL is a safe and effective procedure, which can be considered in selected patients. They insisted that avoiding the use of a nephrostomy tube aids the tamponade to stop bleeding, with the thrombolytic effect of urokinase in the urine. Istanbulluoglu et al. [19] stated that patients with no residual stones are appropriate for a totally tubeless procedure because it precludes second-look endoscopic procedures for the treatment of residual stones.

In this study, we performed modified totally tubeless PNL in patients with stone-free status according to the intraoperative evaluation and no significant perforation or bleeding, and it showed pleasing results in terms of postoperative outcomes and safety. This procedure can avoid the postoperative stent-related symptoms and cystoscopy for double-J stent removal. Moreover, it allows possible access for second-look nephroscopy during $24 \mathrm{~h}$ postoperatively. Early postoperative analgesic requirement and analgesic usage were slightly higher compared with those after tubeless PNL, but the difference was not statistically significant. Several studies have shown that postoperative pain and analgesic requirement are similar between the nephrostomy-free group and the small tube nephrostomy group [20-22]. However, one patient who underwent modified totally tubeless PNL showed spontaneous passage of small stone fragments with colic pain and was readmitted to the hospital and treated conservatively. Based on this experience, in patients who do not have a completely stone-free status at the end of the surgery, placement of a double-J stent instead of an externalized ureteral catheter might be more beneficial.

Our study has several limitations worth noting. The length of hospital stay for all 3 groups in this study is over 5 days, which is longer than other PNL studies. This could be affected not only by surgical outcome but also by patients' preferences. In Korea, patients have a tendency for long hospital stays because of low hospital cost due to the regulation of the National Health Insurance Corporation. We evaluated the postoperative analgesic requirement but not the visual analog scale score for the degree of postoperative pain and stent-related symptoms (e.g. frequency, dysuria) to compare among the groups.

\section{Conclusions}

Tubeless PNL and modified totally tubeless PNL are comparable to the standard PNL in terms of postoperative outcomes and safety and seem to have benefits in terms of length of hospitalization, postoperative pain, and nephrostomy tube site com- 
plications. Modified totally tubeless PNL could avoid stent-related symptoms and postoperative cystoscopy for stent removal, and it could be considered as an effective and safe alternative treatment option for treating renal and upper ureteral stones in selected patients.

\section{Conflict of interest}

The authors declare no conflict of interest.

\section{References}

1. Fernström I, Johannson B. Percutaneous pyelolithotomy. A new extraction technique. Scand J Urol Nephrol 1976; 10: 257-9.

2. Pietrow PK, Auge BK, Lallas CD, et al. Pain after percutaneous nephrolithotomy: impact of nephrostomy tube size. J Endourol 2003; 17: 411-4

3. Shah HN, Kausık VB, Hegde SS, et al. Tubeless percutaneous nephrolithotomy: a prospective feasibility study and review of previous reports. BJU Int 2005; 96: 879-83.

4. Rana AM, Mithani S. Tubeless percutaneous nephrolithotomy: call of the day. J Endourol 2007; 21: 169-72.

5. Mouracade P, Spie R, Lang H, et al. Tubeless percutaneous nephrolithotomy: what about replacing the Double-J stent with a ureteral catheter? J Endourol 2008; 22: 273-5.

6. Crook TJ, Lockyer CR, Keoghane SR, et al. Totally tubeless percutaneous nephrolithotomy. J Endourol 2008; 22: 267-72.

7. Crook TJ, Lockyer CR, Keoghane SR, et al. A randomized controlled trial of nephrostomy placement versus tubeless percutaneous nephrolithotomy. J Urol 2008; 180: 612-4.

8. Borges CF, Fregonesi A, Silva DC, et al. Systematic review and meta-analysis of nephrostomy placement versus tubeless percutaneous nephrolithotomy. J Endourol 2010; 24: 1739-46.

9. Goodwin WE, Casey WC, Woolf W. Percutaneous trocar (needle) nephrostomy in hydronephrosis. J Am Med Assoc 1955; 157: 891-4.

10. Wickham JE, Miller RA, Kellett MJ, et al. Percutaneous nephrolithotomy: one stage or two? Br J Urol 1984; 56: 582-5.

11. Bellman GC, Davidoff R, Candela J, et al. Tubeless percutaneous renal surgery. J Urol 1997; 157: 1578-82.

12. Limb J, Bellman GC. Tubeless percutaneous renal surgery: review of first 112 patients. Urology 2002; 59: 527-31.

13. Delnay KM, Wake RW. Safety and efficacy of tubeless percutaneous nephrostolithotomy. World J Urol 1998; 16: 375-7.

14. Desai MR, Kukreja RA, Desai MM, et al. A prospective randomized comparison of type of nephrostomy drainage following percutaneous nephrostolithotomy: large bore versus small bore versus tubeless. J Urol 2004; 172: 565-7.

15. Agrawal MS, Agrawal M, Gupta A, et al. A randomized comparison of tubeless and standard percutaneous nephrolithotomy. J Endourol 2008; 22: 439-42.

16. Al-Ba’adani HT, Al-Kohlany KM, Al-Adimi A, et al. Tubeless percutaneous neprolithotomy: the new gold standard. Int Urol Nephrol 2008; 40: 603-8.
17. Agrawal MS, Sharma M, Agarwal K. Tubeless Percutaneous nephrolithotomy using antegrade tether: a randomized study. J Endourol 2014; 28: 644-8.

18. Karami H, Gholamrezaie HR. Totally tubeless percutaneous nephrolithotomy in selected cases. J Endourol 2004; 18: 475-6.

19. Istanbulluoglu MO, Cicek T, Ozturk B, et al. Percutaneous nephrolithotomy: nephrostomy or tubeless or totally tubeless? Urology 2010; 75: 1043-6.

20. Choi M, Brusky J, Weaver J, et al. Randomized trial comparing modified tubeless percutaneous nephrolithotomy with tailed stent with percutaneous nephrostomy with smallbore tube. J Endourol 2006; 20: 766-70.

21. Shah HN, Sodha HS, Khandkar AA, et al. A randomized trial evaluating type of nephrostomy drainage after percutaneous nephrolithotomy: small bore $v$ tubeless. J Endourol 2008; 22: 1433-9.

22. Shen P, Liu Y, Wang J. Nephrostomy tube-free versus nephrostomy tube for renal drainage after percutaneous nephrolithotomy: a systematic review and meta-analysis. Urol Int 2012; 88: 298-306.

Received: 8.08.2016, accepted: 23.11.2016. 